EPJ Web of Conferences 59, 14004 (2013)

DOI: $10.1051 /$ epjconf/20135914004

(C) Owned by the authors, published by EDP Sciences, 2013

\title{
Influence of the number of atomic levels on the spectral opacity of low temperature nickel and iron in the spectral range $50-300 \mathrm{eV}$
}

\author{
M. Busquet ${ }^{1, a}$, M. Klapisch ${ }^{1}$ and D. Gilles ${ }^{2}$ \\ 1 ARTEP Ellicott City, MD 21042, USA \\ ${ }^{2}$ CEA/IRFU/Sap, 91191 Gif-sur-Yvette Cedex, France
}

\begin{abstract}
Opacity is a fundamental ingredient for the secular evolution of stars. The calculation of the stellar plasma absorption coefficients is complex due to the composition of these plasmas, generally an $\mathrm{H} / \mathrm{He}$ dominated mixture with a low concentration of partially ionized heavy ions (the iron group). The international collaboration OPAC recently presented extensive comparisons of spectral opacities of iron and nickel for temperatures between 15 and $40 \mathrm{eV}$ and for densities of $\sim 3 \mathrm{mg} / \mathrm{cm}^{3}$, relevant to the stellar envelope conditions $[1,2]$. The role of Configuration Interaction (CI) and the influence of the number of atomic levels on the opacity using the recently improved version of HULLAC atomic code $[3,4]$ are illustrated in this article. Comparisons with theoretical predictions already presented in [1] are discussed.
\end{abstract}

\section{HULLAC AND HULLAC-V9 ATOMIC CODES}

HULLAC is an atomic structure code, in the relativistic and $\mathrm{j}-\mathrm{j}$ coupling framework. It solves selfconsistently the Dirac equation in a central potential. Wave functions, level energies are computed but also the cross sections for the 5 processes (radiative spontaneous decay, auto-ionization, collisional excitation, radiative and collisional recombination). Use of parametric potential [3] is preferred to the Dirac-Fock method, angular algebra is solved with the NJGRAF package and collisional transitions use the Distorted Wave approximation. Phase-Amplitude and Factorization-Interpolation methods are in use and allow dramatic reduction of the computation time.

HULLAC-v9 [4] is a major evolution of the HULLAC code developed by A. Bar-Shalom, J. Oreg and M. Klapisch [3]. Users will benefit of the new "modes" (like the direct configuration average mode "C", or the CI in restricted groups of levels), of the versatile output format and the general ease of use (as for example automatic generation of configurations, like using expression $3 \mathrm{~L}^{\wedge} \mathrm{N}$ to define all configurations with $\mathrm{N}$ electrons in $\mathrm{M}$ shell $\mathrm{n}=3$ ). This is illustrated in Fig. 1, which explains how to generate superconfigurations in HULLAC code. HULLAC-v9 includes a Collisional-Radiative Model solver, able to deal with hundreds of thousands states, thanks to an original pre-conditioned sparse matrix solver. Figure 1 also shows an example of the effect of the number of levels included in the $\mathrm{Ni}$ opacity calculation for the conditions specified on the figure: ground state of Ni VII ion ("G", with 2286 levels, dotted red line), ground state plus some excited levels of Ni VII ion ("G3”, black line with some features), $\Delta \mathrm{n}=0, \mathrm{n}=3$ contributions from dominant Ni ions ("total" dashed blue line with 71833 levels including 7256 levels for only Ni VII $(\Delta n=0, n=3)$ ion contributions. Computation of half a billion lines are now possible. This can seem large but even such large numbers are not enough to treat the complexity of the spectrum we are interested in, as we will see on a nickel example for

\footnotetext{
ae-mail: busquet@artepinc.com
}

This is an Open Access article distributed under the terms of the Creative Commons Attribution License 2.0, which permits unrestricted use, distribution, and reproduction in any medium, provided the original work is properly cited. 
(a)

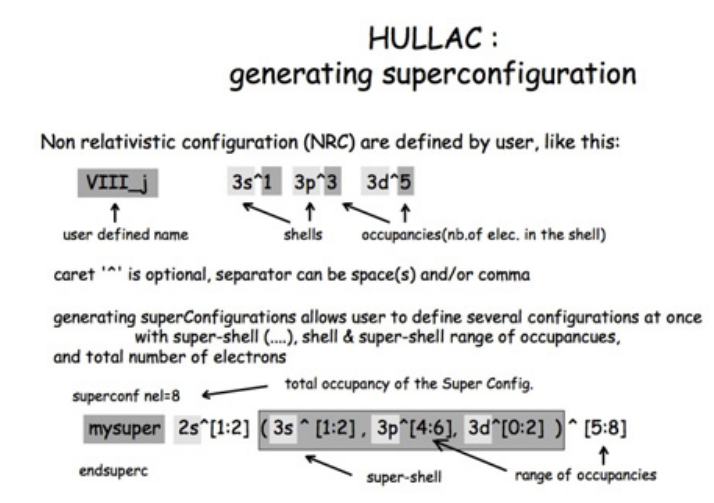

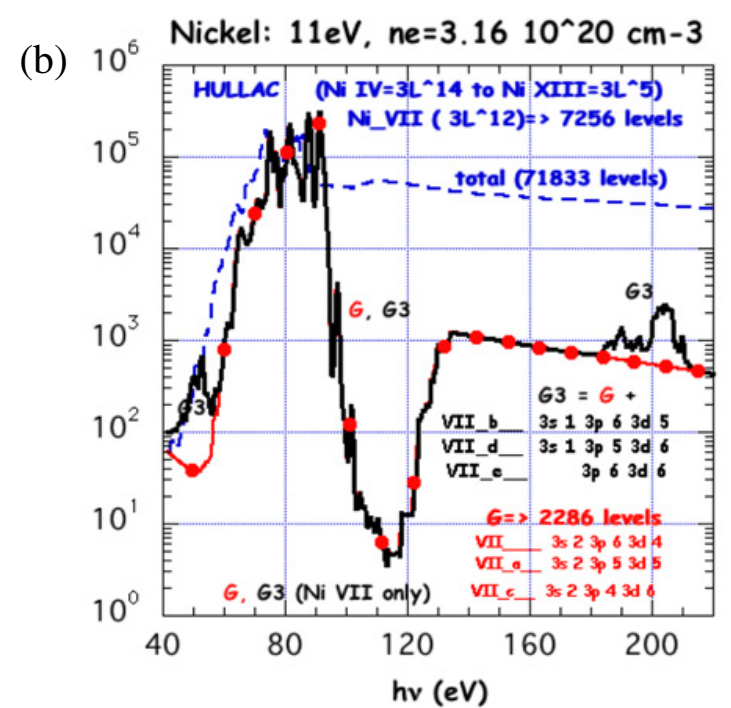

Figure 1. a) This panel explains how to generate superconfigurations in HULLAC code. b) Example of the effect of the number of levels included in the Ni opacity calculation.

low densities and temperatures around $15 \mathrm{eV}$. The full $\mathrm{CI}$ treatment involves also a large number of coefficients (NEGVEC), which can be so large that calculation for complex systems can be hardly tractable.

\section{CONFIGURATION INTERACTION (CI) TREATMENT IN HULLAC-V9}

Figure 2 illustrates two examples of the effect of CI modes on the opacity result (nickel and iron), mostly a notable shift in energy of the peak of the spectrum (dashed black line for full CI, green and blue lines for submode $\mathrm{C}$ and $\mathrm{L} \_$noCI). Only $\Delta \mathrm{n}=0, \mathrm{n}=3$ transitions are included in Fig. 2a. Figure $2 \mathrm{~b}$ shows the combined effect of the submodes, of the number of levels included in the calculation, and of the contributions of the dominant ions for iron at $\mathrm{T}=25 \mathrm{eV}$ and $\rho=8 \mathrm{mg} / \mathrm{cm}^{3}$ (conditions discussed in [5]). Dotted red curve corresponds to full CI mode, including $n=3 \rightarrow n=4$ ("3L $-4 \mathrm{~L}$ ") transitions (Ni VII, 91,944 levels, NEGVEC $=159,742,731),($ Ni VIII, 42,660 levels, NEGVEC $=28,017,451)$, $($ Ni IX, 39,274 levels, NEGVEC $=18,325,729)$, (Ni VIII, 28,906 levels, NEGVEC $=9,629,517)$. In this example ClinLAYZER submode gives results very similar to full CI mode, with much less computation time (green and blue line with triangles).

\section{INFLUENCE OF THE NUMBER OF ATOMIC LEVELS AND CI TREATMENT}

\subsection{Influence of the number of transitions}

Unexplained differences $(\mathrm{h} v>60 \mathrm{eV})$ already discussed in $[1,2]$ for nickel and temperatures around $15 \mathrm{eV}$ between several atomic code results and OP [7] results seem to be due to the insufficient number of transitions for the dominant ionization stages, as it is very difficult to carry transitions involved for these low temperature and spectral range conditions. The role of the $(\Delta \mathrm{n}=0, \mathrm{n}=3)$ transitions of energy above $100 \mathrm{eV}$ is pointed out in this comparison.

In Fig. 3a HULLAC-v9 calculation ("HULLAC", dashed black line) is compared to STA [8] and OP monochromatic opacity results versus energy. Here only STA is reported for clarity as it acts as a fit of 

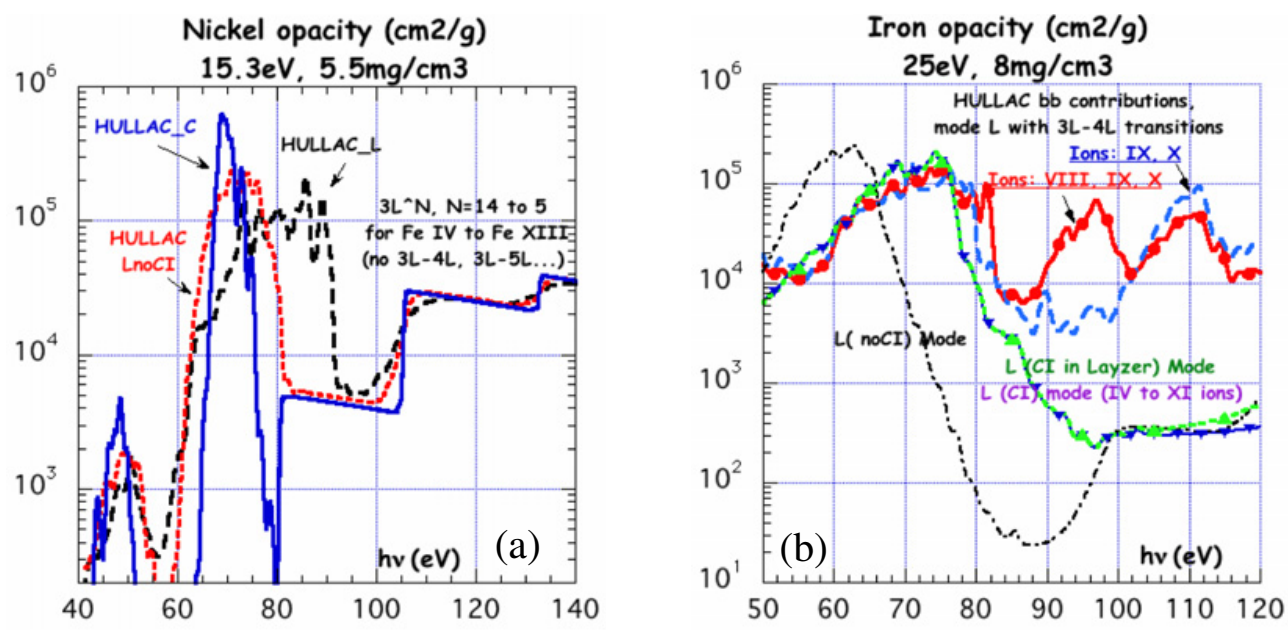

Figure 2. a) Effect of the CI modes on the Ni opacity - mostly a shift in energy. b) Combined effect of the mode and of the influence of the number of levels due to the dominant ions on the iron opacity. Thermodynamical conditions and materials are recalled in the figure.
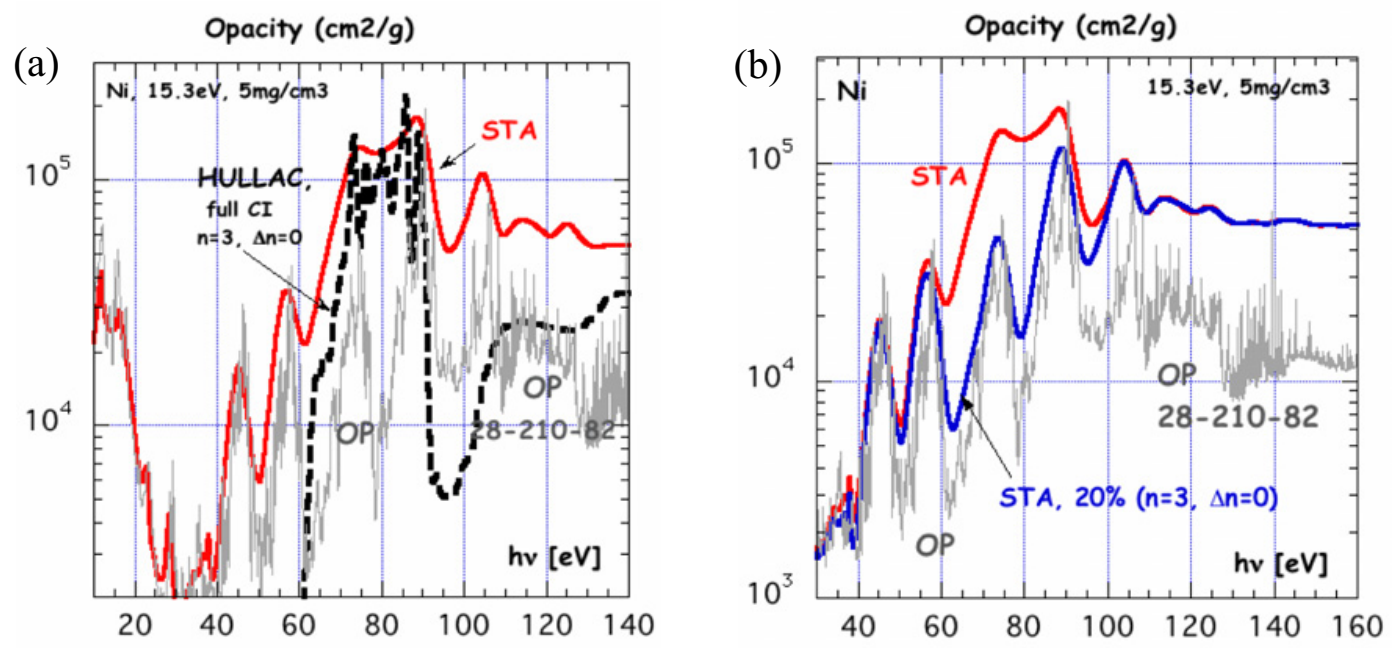

Figure 3. a) Good agreement between HULLAC-v9 and STA Nickel opacity results versus energy for $\mathrm{n}=3$, $\Delta \mathrm{n}=0$ transitions and disagreement with OP database results at low temperature and for energies above $90 \mathrm{eV}$. b) Comparisons between 2 calculations performed with STA code: all $n=3, \Delta n=0$ transitions with computed intensities (red) or divided by 5 (blue) and OP opacity code results.

the other results for these conditions $[1,2,6]$. STA codes account for CI restricted to levels in one non relativistic configuration. The agreement is good between HULLAC-v9 and STA results in the region of interest (only full CI $\Delta \mathrm{n}=0, \mathrm{n}=3$ have been included in HULLAC-v9) but this is not the case for OP results as observed in Fig. 3a, between 70 and $90 \mathrm{eV}$. It is surprising that to recover OP results, in this spectral range, we have to divide by 5 the STA lines $(\Delta n=0, n=3)$, as it can be seen in Fig. 3b. Above $100 \mathrm{eV}$ other transitions are also involved and will be discussed later. Nevertheless OP results are in agreement with STA and other codes for lower energies, the corresponding transitions are not included in the HULLAC-v9 curve displayed in Fig. 3a for clarity. 


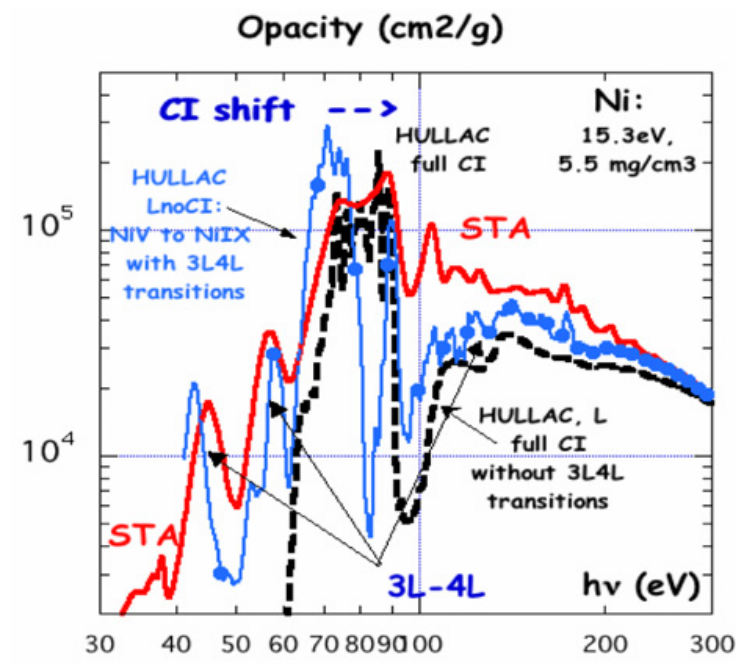

Figure 4. Importance of the 3L-4L contributions to the nickel opacity energy spectral calculations and comparison with STA results. CI produces a large shift of the M shell transitions.

\subsection{Inclusion of 3L-4L transitions with configuration interaction}

Figure 4 illustrates the importance and the difficulty of the inclusion of Configuration Interaction together with taking into account all $3 \mathrm{~L}-4 \mathrm{~L}$ transitions (of dominant ionization stages) at $\mathrm{T}=15.3 \mathrm{eV}$ and $\rho=5.5 \mathrm{mg} / \mathrm{cm}^{3}$. Though we have succeeded for low temperature iron opacity (Fig. 2b), HULLACv9 mode L with CI and 3L-4L transitions for nickel are difficult to carry (the number of lines and of CI coefficients leads to a dramatic increase of the computation time) and the work is still in progress. Nevertheless we have reported in Fig. 4 the L_noCI submode calculation including the 3L-4L contributions (blue thin line). The thin arrows are pointing on the $3 \mathrm{~L}-4 \mathrm{~L}$ peaks. The sensitivity of the opacity to $\mathrm{CI}$ in the region $70-90 \mathrm{eV}$ is really large. The dashed arrow points the large resulting shift between full CI (HULLAC L, dashed back line) and no CI. More transitions will certainly be necessary to fill the gap between HULLAC-v9 and STA results (3L-5L, 4L-5L), which account for ClinNRC submode (plain red line) [6]. This work is in progress. However even if all transitions are not yet included in full CI mode in HULLAC-v9 the major contributions have been included and the asymptotic behavior of the opacity is in agreement with STA results. Ni and Fe databases for temperatures between 10 and $40 \mathrm{eV}$ and density between 1 and $10 \mathrm{mg} / \mathrm{cm}^{3}$ are under construction.

\section{References}

[1] D. Gilles, S. Turck-Chièze, G.Loisel \& al, HEDP 7, 312 (2011); S. Turck-Chièze, G. Loisel, D. Gilles \& al, Journal of Physics: Conference Series, 271(1), 012035 (2011)

[2] G. Loisel et al. HEDP 5, 173 (2009); G. Loisel Ph. D (2011)

[3] "HULLAC", A. Bar-Shalom, M. Klapisch, J. Oreg, JQSRT, 71, 169 (2001)

[4] "A New Improved Version of HULLAC", M. Busquet .\& al, J.Phys. IV, 133, 973 (2006); ibid. AIP Conference V, 926, 206 (2009)

[5] D.S. Whittaker, M.H. Edwards and G.J. Tallents, HEDP, 3, 314 (2007)

[6] "Iron and Nickel spectral opacity calculations calculations ... for pulsating stellar envelops...", D. Gilles, S. Turck-Chièze, M. Busquet, F. Thais \& al, these proceedings

[7] N.R. Badnell, M.A. Bautista, K. Butler, F. Delahaye, C. Mendoza, P. Palmeri, C.J. Zeippen and M.J. Seaton Mon. Not. R. Astr. Soc., 360, 458 (2005); “OP”: http://cdsweb.u-strasbg.fr/topbase/

[8] A. Bar-Shalom, J. Oreg, M. Klapisch \& al, Phys. Rev. E, 59, 3512 (1999) 